\title{
OPEN Isolation and characterization of novel primary cells from the human distal outflow pathway
}

\author{
Uttio Roy Chowdhury $\bowtie$, Cindy K. Bahler, Cheryl R. Hann, Bradley H. Holman \& \\ Michael P. Fautsch
}

Ocular hypertension occurs due to increased resistance to aqueous humor removal through the conventional outflow pathway. Unlike the proximal region of the conventional outflow pathway, the distal region has not been well studied, mostly due to lack of model systems. Here we describe isolation and characterization of human primary vascular distal outflow pathway (VDOP) cells from the distal region of the conventional outflow pathway. Tissue from the distal region was isolated from human corneo-scleral rims, digested with collagenase type I $(100 \mathrm{U} / \mathrm{ml})$ and placed on gelatin coated plates to allow cellular growth in Dulbecco's Modified Eagle's Medium (low glucose) containing fetal bovine serum and antibiotic/antimycotic. VDOP cells showed consistent proliferation for up to 7 passages, retained endothelial-like nature of the parent tissues and showed a unique marker phenotype of Lectin ${ }^{+}$VEGFR2-CD34-NG2- that was distinct from neighboring trabecular meshwork (Lectin+VEGFR2-CD34-NG2+) and Schlemm's canal (Lectin+VEGFR2+CD34+'NG2+) cells. Dexamethasone treated VDOP cells did not express myocilin and did not form cross-linked actin networks, in contrast to trabecular meshwork cells. These data show that VDOP cells are unique to the distal outflow region and can be used as a viable in vitro model system to understand the biology of the distal outflow pathway and intraocular pressure regulation.

Aqueous humor, the primary fluid nourishing the avascular tissues of the anterior chamber of eyes, is produced from the epithelium of the ciliary body and exits the anterior chamber through one of two pathways. The conventional (trabecular) pathway, comprising the trabecular meshwork (TM), Schlemm's canal (SC), collector channels and aqueous veins, is the primary pressure-dependent pathway for removing aqueous humor in humans. A secondary route called the uveoscleral outflow pathway is pressure-independent where aqueous humor is removed through the interstitial spaces of the ciliary muscles ${ }^{1}$. A major portion of intraocular pressure in the anterior chamber is generated due to homeostatic resistance to aqueous humor passage at the interface between the proximal region of the TM and inner wall of $S^{1,2}$. Reduction of aqueous humor flow across the TM and SC interface is recognized as the primary cause of elevated intraocular pressure in ocular hypertensive diseases like glaucoma ${ }^{3-7}$.

While the complete molecular events leading to pathological changes in outflow resistance is not understood, there is a wealth of data from in vitro cell culture models of primary normal trabecular meshwork (NTM) and SC cells that have helped describe cellular and molecular events that are altered in normal and ocular hypertensive conditions. For example, primary NTM cells, initially described by Polansky et al. in 1979, have been utilized to characterize their unique properties such as phagocytosis, expression and upregulation of myocilin in response to glucocorticoids, and development of cross-linked actin networks (CLANs) ${ }^{8-13}$. Similarly, establishment of primary cultures of SC cells ${ }^{14}$ have helped understand how changes in physical characteristics of these cells (e.g. stiffness) contribute towards increased resistance to fluid flow ${ }^{15}$.

In addition to outflow resistance generated at the TM and SC interface, evidence also suggests that the distal region of the conventional outflow pathway (region immediately downstream to the outer wall of SC comprising the deep scleral, intrascleral and episcleral plexus regions, referred to as the distal outflow pathway in this article) has a significant role in regulating outflow resistance ${ }^{16-18}$. Even after complete removal of the TM area, human eyes can still retain up to $50 \%$ resistance to outflow ${ }^{17-23}$. Surgical techniques that remove parts of the 


\begin{tabular}{|l|l|l|l|}
\hline VDOP cell line numbers & Age & Gender & Race \\
\hline VDOP2 & 74 & Female & Caucasian \\
\hline VDOP3 & 88 & Female & Caucasian \\
\hline VDOP4 & 52 & Male & American Indian/Alaskan native \\
\hline VDOP5 & 82 & Male & Caucasian \\
\hline VDOP7 & 35 & Male & Caucasian \\
\hline VDOP8 & 20 & Male & Undisclosed \\
\hline VDOP9 & 50 & Male & Caucasian \\
\hline VDOP10 & 7 & Female & Undisclosed \\
\hline NTM cell line numbers & \multicolumn{5}{|l}{} \\
\hline NTM7 & 2 & Female & Undisclosed \\
\hline NTM14 & 32 & Female & Undisclosed \\
\hline NTM15 & 24 & Male & Undisclosed \\
\hline SC cell line numbers & \multicolumn{5}{|l}{} \\
\hline SC76 & 59 & Female & Undisclosed \\
\hline SC78 & 77 & Male & Caucasian \\
\hline SC89 & 68 & Male & Caucasian \\
\hline
\end{tabular}

Table 1. Demographic information of human donors of VDOP, NTM and SC primary cell lines.

distal outflow pathway while maintaining the integrity of the TM, cause similar increases to outflow facility as with removal of TM alone ${ }^{24-27}$. The pulsatile flow of aqueous humor in the distal outflow pathway is lost during glaucoma with a direct correlation between the loss of pulsatile flow to the severity of glaucoma ${ }^{28-31}$. Therefore, targeting the distal outflow pathway can be a viable mode of treatment for ocular hypertensive pathologies like glaucoma that involve perturbations of outflow resistance. However, the distal outflow pathway has not been studied in detail, mostly due to lack of model systems.

Given the importance of the distal outflow pathway in regulating IOP, understanding the biology and functional characteristics of this area is of great clinical relevance. Unfortunately, unlike primary NTM and SC cells, there are no established in vitro cell-based model systems that can be used to study the distal outflow pathway. To address this unmet need, we have isolated and characterized a specific population of vascular endothelial cells that retain and simulate the characteristics of the vessels of the distal outflow pathway. These vascular distal outflow pathway (VDOP) cells are phenotypically and functionally distinct from NTM and SC cells and represent an in vitro model system for understanding the biology of the distal outflow pathway of human eyes.

\section{Results}

Culture and proliferation of VDOP cells. Demographics of the human donor eyes used to obtain primary cells are found in Table 1 . To isolate VDOP cells from the distal outflow pathway of human anterior segments, the TM was removed after making vertical cuts anterior to Schwalbe's line and posterior to the scleral spur and above the outer wall of SC, as previously described ${ }^{13}$. Once the TM was removed, an iris scalpel was used to repeatedly scrape and remove any remaining TM tissue as well as the inner and outer layer of SC. Cells were also scraped from the outside of the eye directly above the episcleral vasculature. Tissue distal to SC was isolated, treated with collagenase, and placed in gelatin-coated 6 well plates and incubated with Dulbecco's modified eagle's media (DMEM; Fig. 1). Cells migrated from the tissue (Fig. 2a,b) to the plate surface and reached 80-90\% confluence within 8-10 days. Cells were transferred to a T75 tissue culture flask at seeding densities of $10^{5}$ cells and referred to as passage 1 . Cells isolated and established from 8 independent human anterior segments showed a consistent doubling time of $48-72 \mathrm{~h}$ over passages $1-7$. Proliferative potential beyond passage 7 was not evaluated.

The overall morphology of VDOP cells exhibiting a distinct flat and cobblestone morphology is consistent with endothelial cells (Fig. 2c). VDOP cells show multiple interlinking processes that can be immunohistochemically stained with antibodies against homing cell adhesion molecule-1 (HCAM-1/CD44) (Fig. 2d). VDOP cells also stained positive for von Willebrand factor, further indicating the endothelial nature of the cells (Fig. 2e).

Phenotypic characterization of VDOP cells. To further evaluate the phenotypic characteristics of VDOP cells ( $n=8$ independent cell lines, 6 at passage 4 , one at passage 5 and one at passage 8), immunohistochemical localization of a panel of endothelial cell surface markers (Table 2) were examined and compared to $\operatorname{NTM}(n=3,2$ at passage 5, one at passage 6$)$ and SC cells $(n=3$, passage 6$)$ to rule out contamination of nearby cells. All cell lines (VDOP, NTM and SC) stained positive for lectin, von Willebrand factor (vWF) and a-smooth muscle actin ( $\alpha$-SMA). Two out of the three SC cell lines tested positive for CD31 (PECAM1) while only one out of 8 VDOP cell lines showed CD31. CD34 was found in SC cells (2 out of 3 lines) but was not present in VDOP or NTMs. When stained for neuronal glial cell 2 (NG2), both NTM and SC cells showed more prevalent NG2 staining compared to VDOP cells (2 out of 3 NTM lines; 3 out of 3 SC lines; 1 out of 8 VDOP lines). However, VDOPs and NTMs were found to be less responsive to VEGFR2 staining (VDOP, 2 out of 8 lines; NTM, 1 out of 3 lines) compared to SC cells (all three lines) (Table 2). Based on percent of independent cell lines that tested 


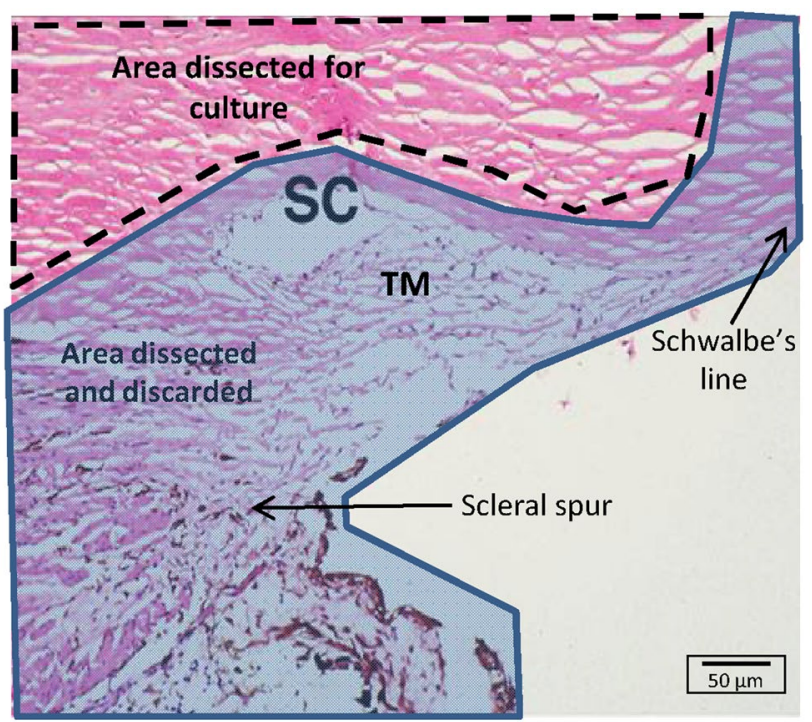

Figure 1. Establishing primary cultures of VDOP cells. Image showing the region of the conventional outflow pathway that was dissected and removed (lightly shaded portions), and the portion isolated (dotted area) for extracting VDOP cells. The extracted tissue rims were cut into 1-2 $\mathrm{mm}$ pieces, incubated in collagenase for 90 min and immersed in low glucose DMEM containing 10\% FBS and 1\% antibiotic/antimycotic on a gelatincoated plate. Scale bar, $50 \mu \mathrm{m}$; SC, Schlemm's canal; TM, trabecular meshwork.
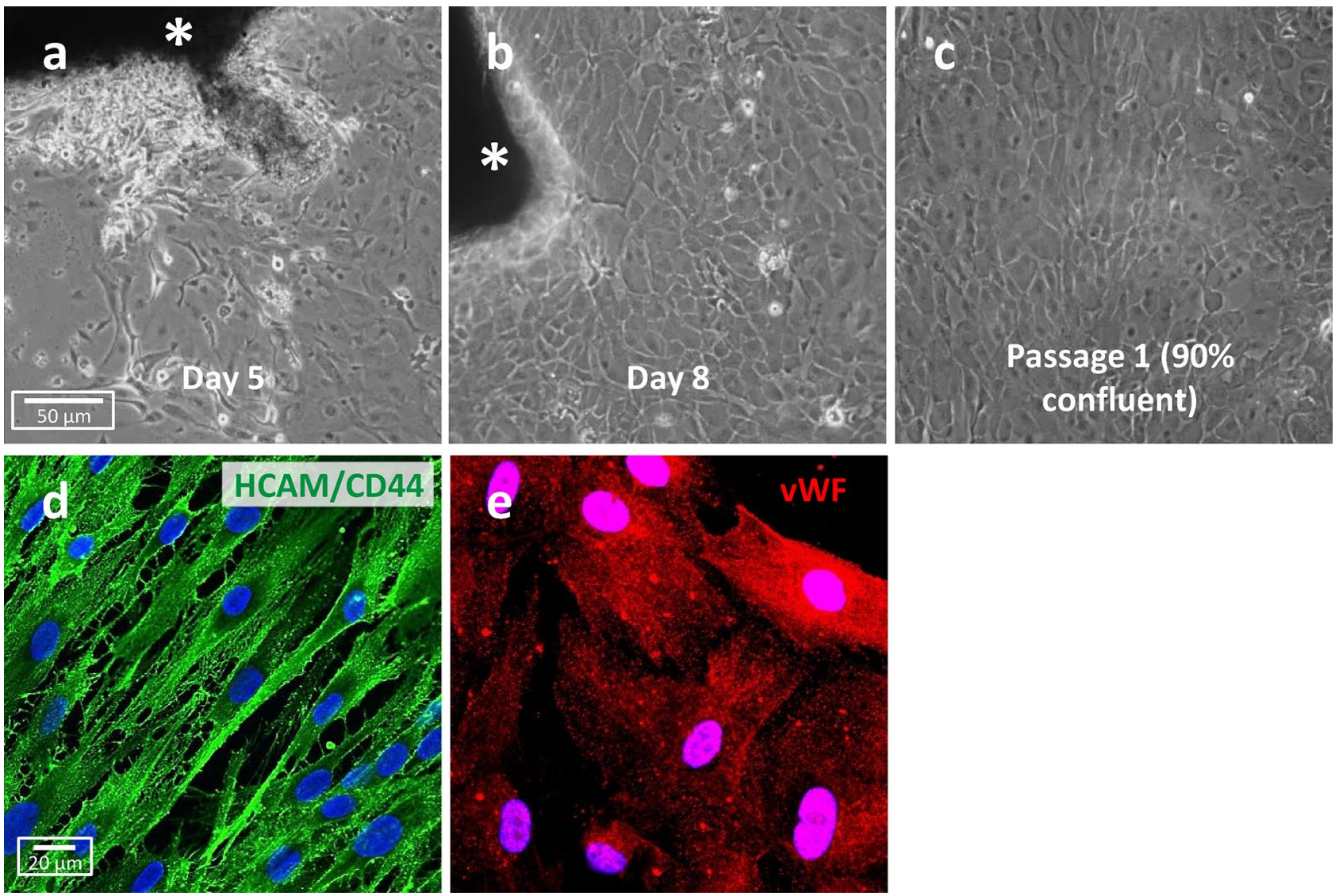

Figure 2. VDOP primary cells. (a, b) Gross morphological characteristics of VDOP cells growing out of tissue removed from the distal outflow region (asterisks). (c) Primary cell line of VDOP cells at $90 \%$ confluence.

(d) VDOP cells stain positive for HCAM/CD44, highlighting the intercellular processes. (e) VDOP cells also show strong localization of von Willebrand factor (vWF), indicating a possible endothelial lineage of the cells. Representative images from VDOP cells at passage 4 . Scale bar, $50 \mu \mathrm{m}(\mathbf{a}-\mathbf{c}) ; 20 \mu \mathrm{m}(\mathbf{d}, \mathbf{e})$. 


\begin{tabular}{|l|l|l|l|l|}
\hline \multirow{2}{*}{ Marker } & \multirow{2}{*}{ \% Positive cells lines } \\
\cline { 4 - 6 } & Marker type & VDOP $(\mathbf{n}=\mathbf{8})$ & NTM $(\mathbf{n}=\mathbf{3})$ & SC $(\mathbf{n}=\mathbf{3})$ \\
\hline Lectin & Endothelial & 100 & 100 & 100 \\
\hline von Willebrand factor (vWF) & Microvessel & 100 & 100 & 100 \\
\hline$\alpha$-smooth muscle actin $(\alpha$-SMA) & Myofibroblasts & 100 & 100 & 100 \\
\hline CD31 (Platelet endothelial cell adhesion molecule, PECAM1) & Microvessel & 12.5 & 0 & 67 \\
\hline CD34 & Stem cell & 0 & 0 & 67 \\
\hline Neuron-glial antigen 2 (NG-2) & Neuronal glial cell-2 & 12.5 & 67 & 100 \\
\hline Vascular endothelial growth factor-2 (VEGFR2) & Endothelial & 25 & 33 & 100 \\
\hline
\end{tabular}

Table 2. Various phenotypic markers and their staining frequencies in VDOP, NTM and SC cell lines.
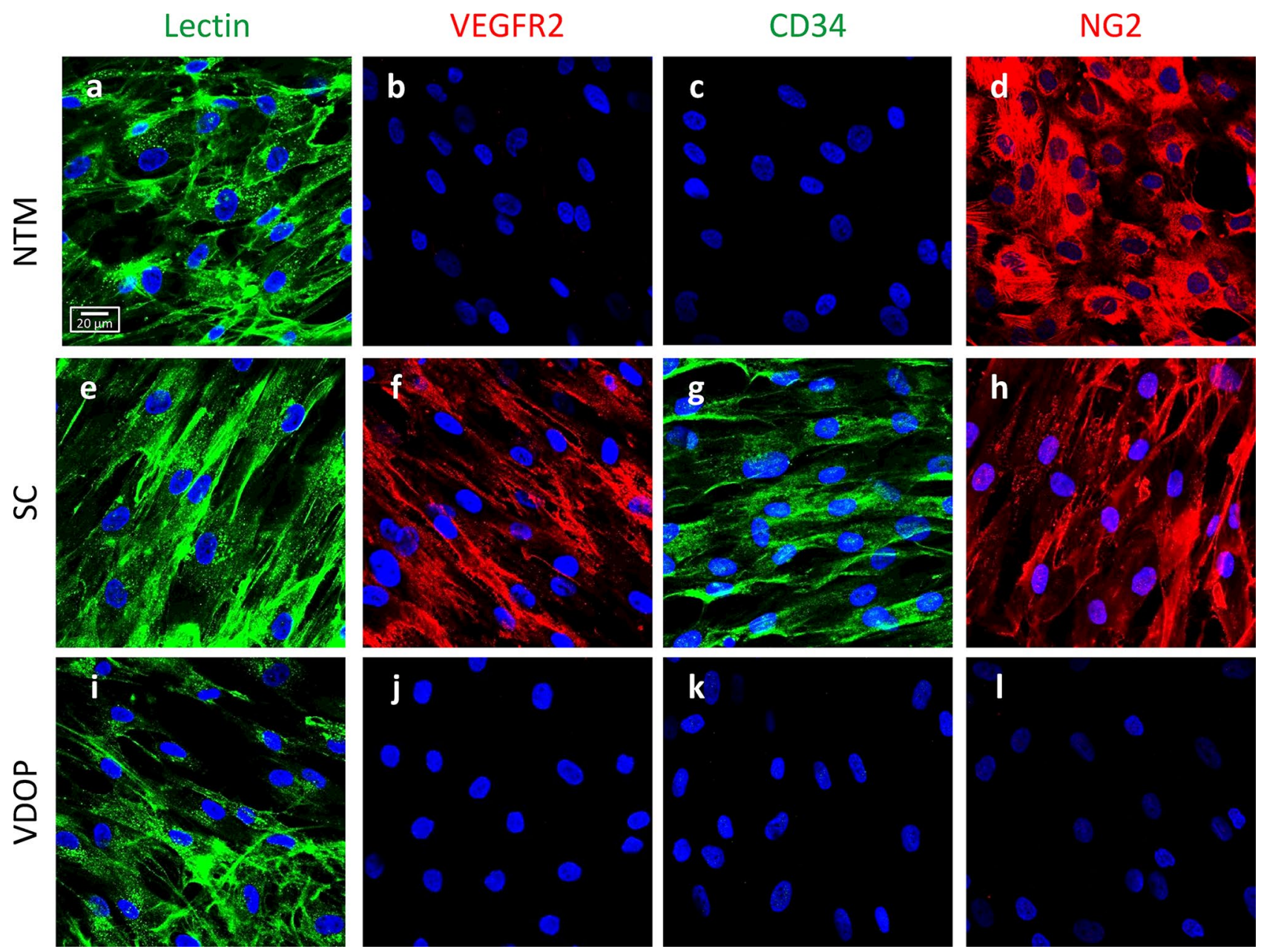

Figure 3. Phenotypic characterization of VDOP cells. Localization of various endothelial markers lectin, VEGFR2, CD34 and NG2 in NTM (a-d), SC (e-h) and VDOP (i-l) cells using immunohistochemistry. All three cell types were positive for Lectin $(\mathbf{a}, \mathbf{e}, \mathbf{i})$ while VEGFR2 was seen only in SC and VDOP cells $(\mathbf{f}, \mathbf{j})$. CD34 was found only in SC cells $(\mathbf{g})$ whereas both NTM and SC cells stained positive for NG2 (d, h). Based on this staining pattern, NTM cells were defined as Lectin ${ }^{+}$VEGFR2-CD34- ${ }^{-}{ }^{-} 2^{+}$, SC cells as Lectin $^{+}$VEGFR2 ${ }^{+} \mathrm{CD} 34^{+} \mathrm{NG}^{+}$and VDOP cells as Lectin ${ }^{+}$VEGFR2 ${ }^{-} \mathrm{CD} 34^{-} \mathrm{NG}^{-}$. A total of 8 VDOP $(6$ at passage 4 and 2 at passage 5), 3 NTM (2 at passage 5, one at passage 6) and 3 SC (all at passage 6) cell lines were used for this experiment. Scale bar, $20 \mu \mathrm{m}$.

positive or negative for any given markers (Table 2), VDOP cells displayed a distinguishing cell marker profile of Lectin ${ }^{+}$VEGFR2 ${ }^{-}$CD34-NG2- which was different from those shown by NTM (Lectin ${ }^{+}$VEGFR2-CD34-NG2+ and SC cells (Lectin ${ }^{+}$VEGFR2 ${ }^{+} \mathrm{CD} 34^{+} \mathrm{NG}^{+}$) (Fig. 3 ).

To determine if the VDOP cells retain and simulate their marker profile found in vivo, we evaluated localization of several positive markers identified on VDOP cells (lectin, $a$-SMA, vWF), in episcleral vessels identified 

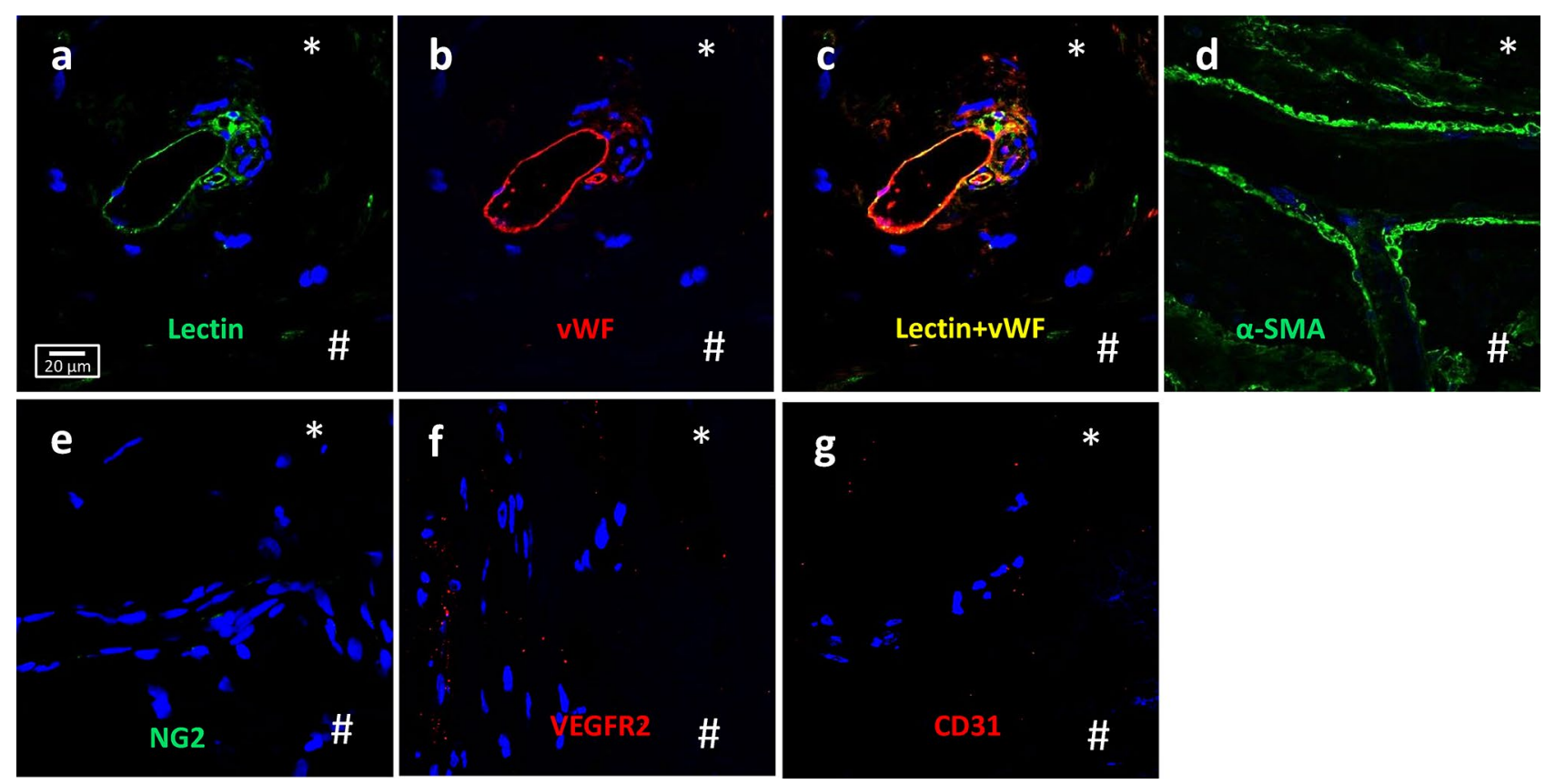

Figure 4. Phenotype characterization of micro-vessels in distal outflow region of human eyes. Representative images showing localization of lectin (a) and $\operatorname{vWF}(\mathbf{b})$ in micro-vessels found in paraffin sections of the distal outflow region (50-100 $\mu \mathrm{m}$ immediately downstream of outer wall of Schlemm's canal and between the outer wall of Schlemm's canal and episcleral region) of the conventional outflow pathway. When superimposed (c), the two molecules showed extensive co-localization in the endothelial cells of the micro-vessel. (d) Vessels from the distal outflow region were also positive for a-SMA but negative for NG2 (e), VEGFR2 (f) and CD31 (g). ${ }^{*}$ egion of the section closest to the episcleral region; \# area closest to the outer wall of Schlemm's canal. Scale bar, $20 \mu \mathrm{m}$.

in sections of the distal outflow region from two independent human donor eyes. Lectin and vWF co-localized in the endothelial cells of the vessels (Fig. 4a-c) and cells surrounding episcleral vessels also stained prominently for a-SMA (Fig. 4d). NG2, VEGFR2 and CD31, which were negative on VDOP cells, were similarly undetectable in the episcleral veins from ocular tissue sections (Fig. 4e-g). These results indicate that the isolated VDOP cells could be true representatives of endothelial cells of the distal outflow region.

Physiological characteristics of VDOP cells. Previous studies have shown that primary NTM cells upregulate myocilin expression and form cross linked actin networks (CLANs) following dexamethasone treatment-physiologic parameters that are unique to these cells ${ }^{8-10}$. Following treatment with dexamethasone $\left(10^{-7} \mathrm{M}, 48 \mathrm{~h}\right)$, VDOP cells $(\mathrm{n}=5)$ did not show any CLAN formation (Fig. $\left.5 \mathrm{a}, \mathrm{b}\right)$ whereas extensive CLANs were noted in NTM cells $(n=2)$ similarly treated with dexamethasone and used as positive controls (Fig. $5 c, d)$. Western blot analysis of cell lysates isolated from VDOP and NTM primary cell cultures following treatment with dexamethasone $\left(10^{-7} \mathrm{M}, 48 \mathrm{~h}\right)$ showed no myocilin protein expression in VDOP cells, in contrast to NTM cells which show myocilin expression and upregulation following treatment (Fig. 5e). These data suggest that there are functional and phenotypic differences between VDOP and NTM primary cell cultures.

Degree of similarity in independent VDOP cell lines isolated from different patients. Due to the primary nature of VDOP cell lines, they need to be repeatedly generated from independent donor eyes post mortem, to maintain a steady supply for experimental procedures. We evaluated whether our standardized technique for isolating these cells is able to select the same kind of cells from various patient samples. We analyzed the transcriptome from three independent VDOP cell lines, each grown in three different culture conditions (50\% human aqueous humor, $10 \%$ fetal bovine serum (FBS), serum-free media) using RNASeq. Principal component analysis of the transcriptome from three cell lines, when reduced to a two dimensional plot, showed a single cluster for all cell lines across all culture conditions (Fig. 6). The high consistency in expressed transcription profiles across cell lines suggests that extraction of VDOP cells from the distal outflow region is consistent between isolates from independent donors.

\section{Discussion}

With the distal outflow pathway gaining importance as a key component of aqueous humor outflow regulation in human eyes, there has been renewed interest in studying this region with the goal of identifying novel therapeutic targets that can be exploited to better treat ocular hypertensive diseases like glaucoma ${ }^{16}$. A major limitation to this strategy has been the lack of appropriate in vitro, cell culture based model systems for this region. Consequently, mechanistic aspects of the region's involvement in outflow regulation are largely unknown. In the current study, we describe the successful isolation and culture of human vascular endothelial-like cells from the distal outflow 


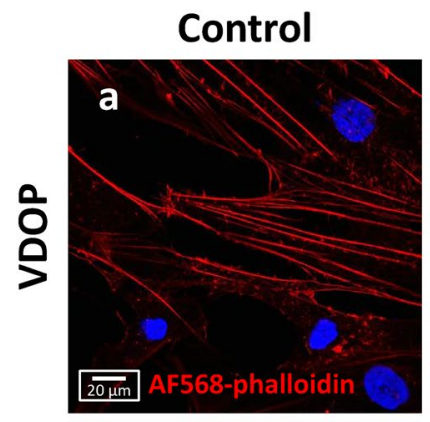

\section{Dexamethasone}
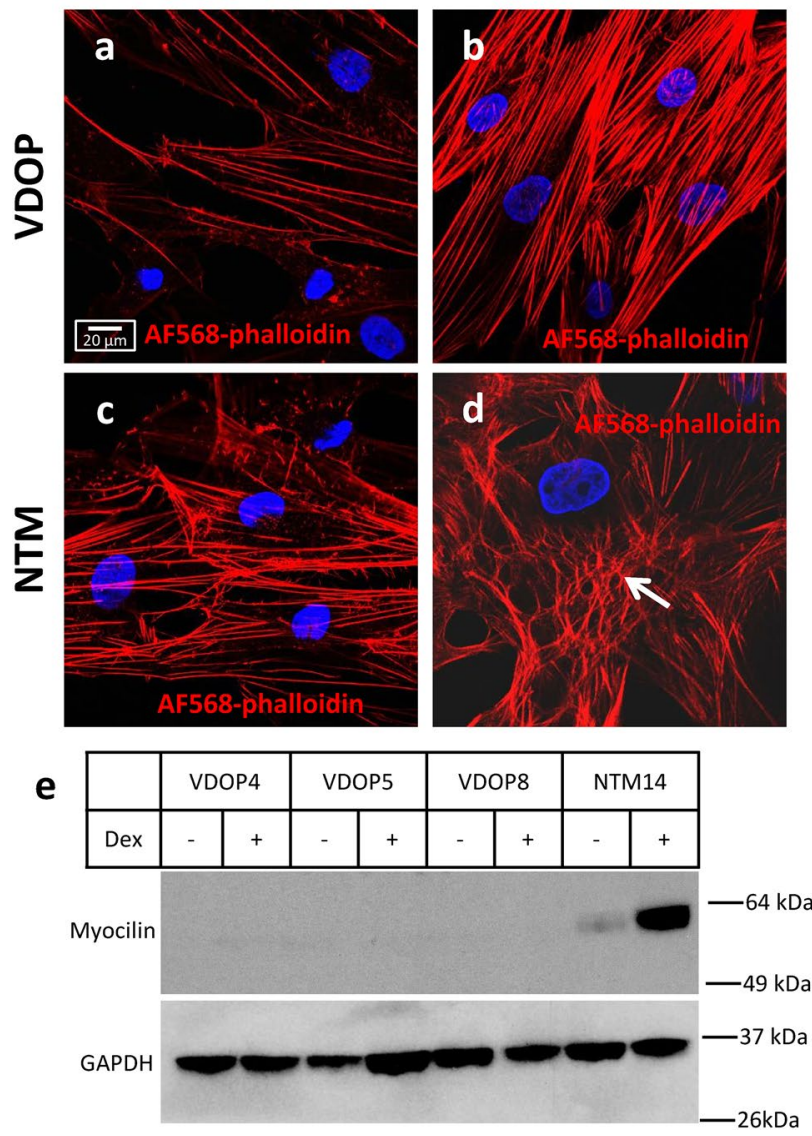

Figure 5. CLAN formation in NTM but not VDOP cell lines. (a, b) Representative confocal images showing complete absence of CLANs in VDOP cells after treatment with dexamethasone for $48 \mathrm{~h}$. (c, d) NTM cells show extensive CLAN formation after similar treatment with dexamethasone (arrow). CLANs were identified by staining the actin cytoskeleton with Alexa Fluor 568 (red) conjugated phalloidin. (e) Expression of myocilin was not detectable in VDOP cells. However human NTM cells showed myocilin expression and upregulation following dexamethasone treatment. GAPDH was used as internal loading control. Full-length blots of myocilin and GAPDH are presented in supplementary Fig. 1a and 1b. Scale bar, $20 \mu \mathrm{m}$.

region of the conventional outflow pathway. These VDOP cells are phenotypically and physiologically distinct from nearby TM and SC cells.

The method for isolating VDOP cells is highly reproducible, with a distinct characteristic marker profile of Lectin ${ }^{+}$VEGFR2 ${ }^{-}$CD $34^{-}{ }^{-N G} 2^{-}$. This signature profile was used to validate each new cell line isolated from independent donor eyes. Additionally, RNASeq studies confirmed that independent VDOP primary cell lines $(n=3)$ had similar transcriptome profiles to each other when cultured in 50\% human aqueous humor, 10\% FBS or in serum-free media. These studies indicate that the initial dissection, cell separation and subsequent cell culture procedures consistently isolate and propagate a unique endothelial-like cell population from the distal outflow region. Due to the primary nature of the VDOP cells, mostly early passage cells (6 of the 8 VDOP lines were used at passage 4 , one at passage 5 and one at passage 8) were used to investigate the marker phenotypes with only one cell line used at passage 8 to evaluate markers in a late passage. Although all cell lines showed similar results, to further validate the markers, we randomly selected two of the early passage VDOP cell lines and probed for select markers at passage 6 and found similar results as reported for passage 4 cells (data not shown). However, this study did not specifically look at the changes in marker profile across passage numbers. Our data only shows that primary cultures of VDOP cells up to passage 8 may be distinguished by the reported marker profile of Lectin ${ }^{+}$VEGFR2 ${ }^{-} \mathrm{CD} 34^{-} \mathrm{NG} 2^{-}$.

Detailed analysis of the characteristics of VDOP cells, isolated from the distal outflow region indicates that they are functionally different from NTM cells. VDOP cells show neither dexamethasone-mediated upregulation of myocilin nor CLAN formation, both physiological responses seen in NTM cells ${ }^{10,13,32}$. These data suggest that VDOP cells are a unique cell population distinct from NTM cells. However, VDOP cells do share several common endothelial markers with NTM (viz. Lectin, vWF, $a-S M A)$. This is not surprising, since cells of the TM are derived from the mesenchyme/neural crest and are endothelial in nature ${ }^{33}$. The endothelial glycoprotein marker lectin has been previously shown to be an important molecule in development of the anterior chamber angle and the $\mathrm{TM}^{34}$. However, unlike NTM cells, VDOP cells did not show expression of NG2, a pericyte/smooth muscle 


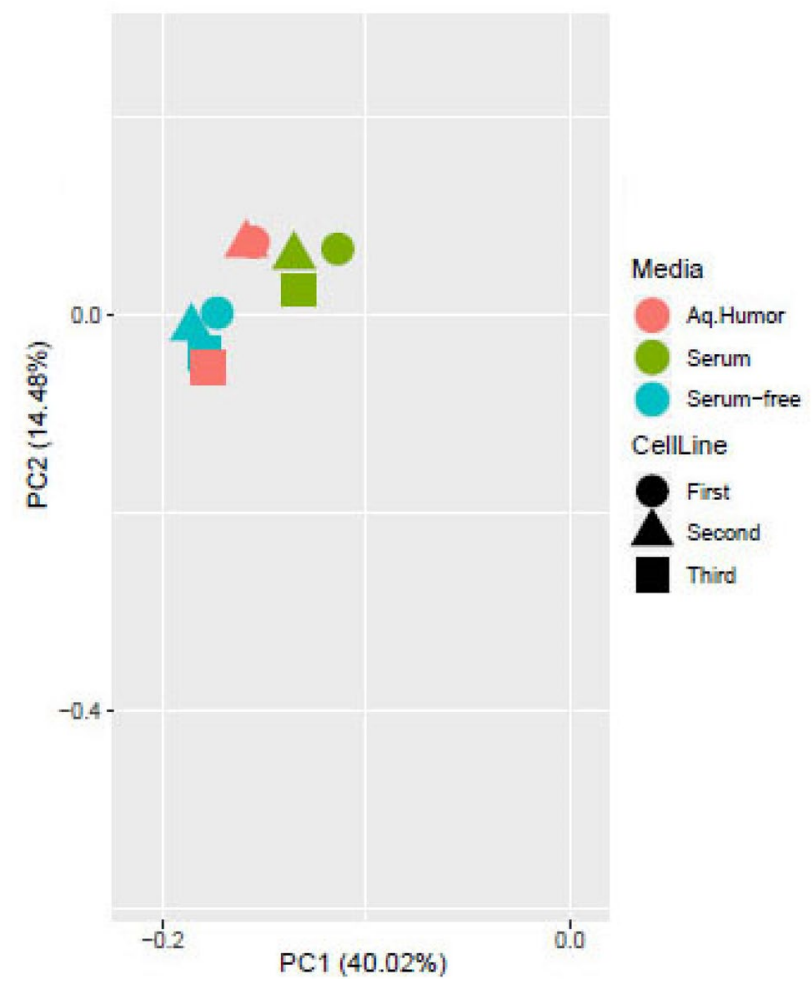

Figure 6. Comparison of independent VDOP cell lines in different media conditions. RNASeq analysis of total RNA from three independent VDOP lines (passage 5), grown in 50\% human aqueous humor, 10\% FBS and serum-free media. Principal component analysis shows closely localized clusters of all 3 cell lines in each media condition indicating high similarity between transcriptomes of the three independent VDOP cell lines.

cell marker. Based on reported contractile properties of cells in the $\mathrm{TM}^{29}$, it is logical that we would see a smooth muscle marker on NTM cells. Overall, the phenotypic marker profiles show that while both NTM and VDOP cells share some common endothelial properties, they are distinct sub classes of endothelial cells.

SC cells originate from the mesodermal germ layer by a unique sequence of vascular development named canalogenesis $^{35}$ and retain their vascular nature even in adult subjects ${ }^{33,36}$. Because of this, SC cells show several

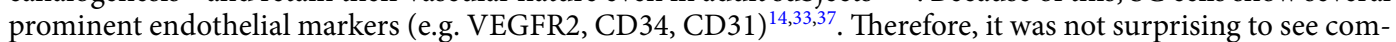
mon endothelial markers such as lectin and vWF between VDOP and SC cells. However, VDOP cells did not show VEGFR2 or CD34, two markers that have been previously shown to be expressed in SC cells ${ }^{33,35}$. Their absence in VDOP cells further indicate that these unique cells are a sub-population of endothelial cells and distinct from SC cells. While VEGFR2 and CD34 are common endothelial/hematopoietic markers, they are expressed more in endothelial cells with strong potential for growth and restructuring and less in more established vascular beds like that of the episcleral plexus ${ }^{35,38,39}$.

Our primary goal for creating the VDOP cell lines was to develop a reproducible in vitro model for studying the involvement of the distal outflow region in regulation of aqueous outflow. While some authors have differentiated this area into deep scleral, intracscleral and episcleral plexus ${ }^{20}$, it is important to note that it was not possible to separate these through dissection. However, cells making up the endothelial-like vessels across the sub regions are similar in nature and the major difference between these regions appears to be in the proximity and complexity of the vascular beds ${ }^{40,41}$. Since VEGFR2, CD34 and NG2 are negative in VDOP cells, we could not use these to validate their presence in vivo in the vessels. For this reason we selected two separate markers viz. aSMA and vWF that were positive in VDOP cells and with lectin, showed that we could indeed find cells that were positive for all three (i.e. $\alpha \mathrm{SMA}^{+} \mathrm{vWF}^{+} \mathrm{Lectin}^{+}$) in the vessels of the distal outflow pathway in vivo. The presence of lectin, vWF and $\alpha$-SMA in both VDOP cells and tissue sections of the distal outflow vessels indicate that the cultured VDOP cells retain the vascular phenotype of their parent tissues and are representative of the distal outflow region.

While we have carefully distinguished VDOP cells from NTM and SC cells, we cannot rule out the possibility that our cultures contained cells from other nearby tissues. The most likely cellular contaminants would be scleral or conjunctival cells. However, scleral cells are mostly terminally differentiated keratinized cells and it is unlikely that these cells would de-differentiate and show proliferative properties similar to what we see in VDOP cells. To prevent entry and growth of conjunctival cells in our cultures, we removed the conjunctiva from the donor eyes, followed by vigorous scraping of the sclera with a scalpel to remove conjunctival remnants prior to dissection. Given that conjunctival cells are more epithelial in nature ${ }^{42}$ whereas VDOP cells are endotheliallike, it seems unlikely that VDOP cells are derived from contamination of conjunctival cells. Furthermore, 
with RNASeq profiles that are nearly identical between different VDOP cell lines, the possibility of a significant contamination is highly unlikely.

There are numerous avenues of research where VDOP cell lines could be useful. For example, evaluating if and how these cells are altered during disease could significantly aid in understanding mechanistic events during ocular hypertensive disorders like glaucoma. VDOP cells may be a valuable in vitro model system to study how current and future ocular hypotensive drugs or other general agents (small molecules, biological compounds, etc.) can and will affect the physiological function of these cells. Additionally, experiments in primary VDOP cells can aid in designing experiments in animal models. With the use of the described methodology, VDOP cells can be utilized as an in vitro model system for researchers to investigate the cell and molecular mechanisms involved in normal and disease processes in the distal outflow region.

\section{Methods}

Isolation and cell culture conditions for VDOP, NTM and SC cells. All human tissues were used as per guidelines of the Declaration of Helsinki and the study was pre-approved by Mayo Clinic Institutional Review Board. Demographic information of human donors used for isolation of VDOP, NTM and SC primary cell lines are provided in Table 1.

VDOP cells. A total of 8 pairs of corneo-scleral rims were used for this study. All eye tissues were free from ocular disorders and malignancies and were supplied by the Lions Gift of Sight (St. Paul, MN) under our preapproved IRB protocol. Informed consent was obtained from the family of the deceased by the Lions Gift of Sight, prior to tissue collection. The tissues were obtained without any patient identifiers, except for the age, gender and general cause of death. Corneo-scleral rims were stored while immersed in Optisol (Numedi, Isanti, $\mathrm{MN}$ ) at $4{ }^{\circ} \mathrm{C}$. All tissues were processed for cell isolation within $48-72 \mathrm{~h}$ of death. During cell isolation, corneoscleral rims were taken out of optisol and washed 3 times with PBS while gently shaking for 1 min between PBS exchanges. Vertical cuts were made anterior to Schwalbe's line and posterior to the scleral spur and the entire TM tissue was removed under a dissecting microscope (SZX16, Olympus, Tokyo, Japan). The area under the TM was vigorously scraped to remove any TM remnants as well as to remove the outer wall of SC. The dorsal surface of the sclera (opposite the TM) was similarly scraped to remove conjunctival cells and the epithelial cells from the top layer of the sclera. Using a curved scissor, a small ring was cut out around the entire corneal rim (minus the cornea) that included the distal portion of the conventional outflow pathway, downstream from SC (Fig. 1; Supplementary Fig. 1). It should be noted that some authors have further characterized the distal portion of the conventional outflow pathway as deep scleral plexus (immediately distal to SC), intracscleral plexus (anterior to deep scleral plexus) and episcleral plexus (region closest to the dorsal portion of the sclera) ${ }^{20,40,41}$. However, it was not technically feasible to manually separate these areas and therefore the entire distal region was utilized for extraction of VDOP cells.

The dissected ring containing the distal region of the conventional outflow pathway was cut into 1-2 mm pieces and rinsed twice in Hank's balanced salt solution (HBSS; with $\mathrm{Ca}^{2+}$ and $\mathrm{Mg}^{2+}$; Mediatech, Manassas, VA). These 1-2 mm tissue pieces from right and left eyes were combined and incubated in collagenase $(100 \mathrm{U} / \mathrm{ml}$ in HBSS with $\mathrm{Ca}^{2+} \mathrm{Mg}^{2+}$ ) (Mediatech) for $90 \mathrm{~min}$ and washed again with HBSS without collagenase $(3 \times 5 \mathrm{~min})$. After the last wash, tissues were centrifuged and distributed to 2 wells of a gelatin coated 6 well tissue culture plate (Biocoat, Corning, Kennebunk, ME) using a wide bore plastic transfer pipette. A sterile $22 \times 22 \mathrm{~mm}$ cover slip (Fisher Scientific, Waltham, MA) was placed on top of the tissue pieces to prevent them from dislodging. The cover slip was submerged under $1 \mathrm{ml}$ of low glucose DMEM (Gibco, Grand Island, NY) containing 10\% FBS (Gibco) and 1\% antibiotic/antimycotic (Sigma-Aldrich, St. Louis, MO) solution. The plate was placed in a humidified cell culture incubator at $37^{\circ} \mathrm{C}$ and $5 \% \mathrm{CO}_{2}$.

Cellular strands extending from tissue pieces would appear within 3-5 days of culture initiation. At 8-10 days, media was removed and cells were washed 3 times with PBS. Trypsin-EDTA (ThermoFisher Scientific, Waltham, MA) was added to each well and plates were incubated at $37^{\circ} \mathrm{C}$ for $3-5 \mathrm{~min}$. This allowed most of the tissues as well as the cells to detach from the plate. The remaining tissue pieces were manually removed by gentle scraping. Tissue pieces and trypsinized cells were transferred to a $15 \mathrm{ml}$ conical tube and washed with PBS twice and centrifuged. The cell pellet was resuspended in DMEM containing 10\% FBS and 1\% antibiotic/antimycotic, and transferred to a single well of a 6 well tissue culture plate (without gelatin coating). Floating tissue pieces were manually removed with forceps and cells were allowed to settle and proliferate. This was considered passage 0 . Once cells reached $90 \%$ confluence $\left(3-5 \times 10^{5}\right.$ cells), they were trypsinized, pelleted by centrifugation, resuspended in DMEM containing 10\% FBS and transferred to a T75 cell culture flask (Corning, Corning, NY). VDOP cells were subsequently split at 1:4 ratios at $90 \%$ confluence. Doubling time was calculated based on the formula $\left[\right.$ Doubling time $=\left\{\right.$ duration $\left.{ }^{\star} \log (2)\right\} /\{\log ($ final concentration $)-\log ($ initial concentration $\left.)\}\right]$. For subsequent experiments, cells beyond passage 5 were not used in functional studies, although the cells were observed to maintain consistent proliferative potential up to passage 7 . Growth properties beyond passage 7 were not evaluated.

NTM cells. Primary cultures of NTM cells $(n=3)$ were established according to previously reported protocols $^{43,44}$. Cells were maintained in low glucose DMEM (Gibco) containing $10 \%$ FBS (Gibco) and $1 \%$ antibiotic/antimycotic (Sigma-Aldrich) in a humidified incubator at $37^{\circ} \mathrm{C}$ with $5 \% \mathrm{CO}_{2}$ and split at 1:4 ratios. All experiments were performed in cells that were cultured for $\leq 7$ passages.

SC cells. SC cell lines $(\mathrm{n}=3)$ were generous gifts from Dr. W. Daniel Stamer (Duke University, Durham, NC). Procedures for establishing primary cultures of SC cells and their characterization have been previously 


\begin{tabular}{|l|l|}
\hline Primary antibodies & Secondary antibodies \\
\hline $\begin{array}{l}\text { FITC-conjugated Lectin, Sigma-Aldrich (St. Louis, MO) } \\
\text { Cat. No. L0401 }\end{array}$ & NA \\
\hline $\begin{array}{l}\text { Rabbit polyclonal, vWF Abcam (Cambridge, MA) } \\
\text { Cat. No. ab6994 }\end{array}$ & $\begin{array}{l}\text { Alexa Fluor (AF) 546 conjugated anti-rabbit, Invitrogen (Carlsbad, } \\
\text { CA) } \\
\text { Cat. No. A11010 }\end{array}$ \\
\hline $\begin{array}{l}\text { Mouse monoclonal a-SMA, Sigma-Aldrich } \\
\text { Cat. No. A2547 }\end{array}$ & $\begin{array}{l}\text { AF 488 conjugated anti-mouse, Invitrogen } \\
\text { Cat. No. A11059 }\end{array}$ \\
\hline $\begin{array}{l}\text { Mouse monoclonal CD31, R\&D Systems (Minneapolis, MN) } \\
\text { Cat. No. BBA7 }\end{array}$ & $\begin{array}{l}\text { AF 488 conjugated anti-mouse, Invitrogen } \\
\text { Cat. No. A11059 }\end{array}$ \\
\hline $\begin{array}{l}\text { Mouse monoclonal CD34, Cell Signaling Technology (Danvers, MA) } \\
\text { Cat. No. 07/2017 }\end{array}$ & $\begin{array}{l}\text { AF 488 conjugated anti-mouse, Invitrogen } \\
\text { Cat. No. A11059 }\end{array}$ \\
\hline $\begin{array}{l}\text { Mouse monoclonal NG-2, Millipore (Billerica, MA) } \\
\text { Cat. No. 05-710 }\end{array}$ & $\begin{array}{l}\text { AF 546 conjugated anti-mouse, Life Technologies (Carlsbad, CA) } \\
\text { Cat. No. A11003 }\end{array}$ \\
\hline $\begin{array}{l}\text { Rabbit monoclonal VEGFR2, Cell Signaling Technology } \\
\text { Cat. No. 08/2017 }\end{array}$ & $\begin{array}{l}\text { AF 546 conjugated anti-rabbit, invitrogen } \\
\text { Cat. No. A11010 }\end{array}$ \\
\hline $\begin{array}{l}\text { Rat monoclonal HCAM, Santa Cruz Biotechnology (Dallas, TX) } \\
\text { Cat. No. sc-18849 }\end{array}$ & $\begin{array}{l}\text { AF488 conjugated anti -rat, Invitrogen } \\
\text { Cat. No. A11006 }\end{array}$ \\
\hline
\end{tabular}

Table 3. List of antibodies and their distribution source.

described $^{14}$. Similar to VDOP and NTM cells, SC cells were grown in low glucose DMEM (Gibco) containing $10 \%$ FBS (Gibco) and 1\% antibiotic/antimycotic (Sigma-Aldrich) and passaged at 1:3 ratios. Only cells $\leq 7$ were used for experiments.

Evaluation of VDOP phenotype using immunohistochemistry (IHC). VDOP, NTM and SC cells were grown to confluence in 8 well chamber slides (Millicell EZ slide, Millipore, Tullagreen, Ireland), fixed in $4 \%$ paraformaldehyde, blocked in $1 \%$ bovine serum albumin (Sigma-Aldrich, St. Louis, MO), and incubated with a panel of primary antibodies (Table 3) overnight. Primary antibodies were removed, chambers washed with PBS $(3 \times 5 \mathrm{~min})$, and incubated with appropriate fluorophore conjugated secondary antibodies (Table 3 ). Following washes in PBS, chamber partitions were removed and slides were mounted under Vectashield mounting medium containing DAPI (Vector Laboratories, Burlingame, CA). Cells were examined under a confocal laser microscope (Zeiss LSM 780; Carl Zeiss, Thornwood, NY) and laser intensity was adjusted based on negative controls where only secondary antibody was added. Cell lines were considered positive for any given marker when $>80 \%$ of the cells stained positive. Out of the 8 VDOP cell lines used for IHC experiments, 6 were used at passage 4 , one at passage 5 and one at passage 8 .

For tissue staining, the distal outflow pathway region was dissected (as described above), fixed in $10 \%$ neutral buffered formalin (Fisher Scientific), dehydrated in increasing ethanol concentrations (75\%, 85\%, 95\% and 100\%) and embedded in paraffin. Paraffin blocks were cut into $5 \mu \mathrm{m}$ sections, mounted on Superfrost/plus glass slides (Fisher Scientific, Pittsburgh, PA) and incubated at $60^{\circ} \mathrm{C}$ for $2 \mathrm{~h}$. Prior to immunohistochemistry, tissue sections were soaked in xylene to remove paraffin and then rehydrated in decreasing ethanol concentrations $(95 \%, 85 \%$, $75 \%$ ). Antigen retrieval was performed by incubating slides in $1 \mathrm{mM}$ EDTA (pH 8.0) at $95^{\circ} \mathrm{C}$ for 30 minutes $^{45}$. Sections were blocked and permeabilized in PBS containing 3\% BSA and $0.1 \%$ TritonX-100, probed with lectin, vWF, and aSMA primary and corresponding secondary antibodies (Table 3), mounted in Vectashield containg DAPI (Vector Laboratories) and imaged by confocal microscopy (described above).

Evaluation of CLAN formation following dexamethasone treatment. CLANs were evaluated by immunohistochemical methods as described above. Briefly, cells were grown to confluence in 8 well chamber slides (Millicell EZ slide, Millipore). The day prior to initiation of experiment, cells were placed in serum-free media. The next day, cells were treated with either $10^{-7} \mathrm{M}$ dexamethasone dissolved in $2 \%$ methanol in serumfree DMEM or vehicle alone for $48 \mathrm{~h}$. Fresh dexamethasone was added after $24 \mathrm{~h}$. Following treatment, cells were washed and incubated for 15 min with Alexa Fluor 568 conjugated phalloidin (Invitrogen, Carlsbad, CA). After final washes in PBS, chamber partitions were removed, cells were mounted in Vectashield containing DAPI (Vector Laboratories), and imaged with a Zeiss LSM 780 confocal microscope (Carl Zeiss). CLANs were identified as geodesic dome like structures engaging either part or whole of the cellular cytoskeleton ${ }^{9,10,46}$ under water corrected $63 \mathrm{X}$ lens.

Western blot analysis of myocilin expression following dexamethasone treatment. NTM and VDOP cells were grown to confluence in 6 well plates. Evening prior to study, cells were washed with PBS and incubated in serum-free media overnight. The following morning, cells were treated with $10^{-7} \mathrm{M}$ dexamethasone for $48 \mathrm{~h}$ with fresh dexamethasone being added for a second time after the first $24 \mathrm{~h}$. Cells were lysed directly in the wells using an ice-cold lysis buffer (50 mM Tris, $\mathrm{pH} 8.0,0.5 \%$ sodium dodecyl sulfate, $0.5 \%$ Triton X-100, $137 \mathrm{mM} \mathrm{NaCl}, 3 \mathrm{mM} \mathrm{KCl}, 8 \mathrm{mM} \mathrm{Na} 2 \mathrm{HPO}_{4}-7 \mathrm{H}_{2} \mathrm{O}, 1 \mathrm{mM} \mathrm{KH}_{2} \mathrm{PO}_{4}$ ) pre mixed with protease and phosphatase inhibitors (Roche, Indianapolis, IN), collected and stored at $-80^{\circ} \mathrm{C}$ until further use.

After analyzing total protein content using Bradford's assay, cell lysates were mixed with $5 \mathrm{X}$ reducing sample buffer (Thermo Scientific, Waltham, MA) containing 15\% $\beta$-mercaptoethanol (Sigma-Aldrich). Equal amounts 
of total protein $(15-20 \mu \mathrm{g})$ was loaded in each well of a 4-15\% gradient SDS-PAGE gel (Bio-Rad, Hercules, CA), transferred to polyvinylidene difluoride membranes (Millipore, Billerica, MA) and blocked in 2\% non-fat dried milk, as described previously ${ }^{43,47}$. Once protein transfer was complete, blots were probed with primary antibodies against rabbit anti-human polyclonal myocilin (developed in our laboratory against the peptide sequence ARPQETQEGLQRELGTLRRERDQLC) ${ }^{48}$ overnight. Following incubation, blots were washed with Tris-buffered saline containing Tween 20 (20 mM Tris [pH 7.5], $150 \mathrm{mM} \mathrm{NaCl}, 0.05 \%$ Tween-20) and incubated with horseradish peroxidase conjugated anti-rabbit secondary antibody (GE Healthcare, Piscataway, NJ). Protein bands were visualized with ECL western blot signal detection reagent (GE Healthcare) and Kodak Biomax XAR films (Eastman Kodak, Rochester, NY). After probing for myocilin, membranes were stripped with 5 M guanidine hydrochloride (Sigma-Aldrich), washed in deionized water and reprobed with mouse anti-human GAPDH (Novus Biologicals, Littleton, CO) which was utilized as loading control, followed by horse-radish peroxidase conjugated anti-mouse secondary antibody (GE Healthcare).

RNA sequencing. Total RNA was isolated from three independent confluent VDOP cell lines using RNeasy mini kit (Qiagen, Valencia, CA) following the manufacturer's protocol. An RNA library was prepared for each cell line using the TruSeq RNA sample Prep kit version 2 (Illumina, San Diego, CA, USA). In samples with RNA integrity number $>7.0$, ribosomal transcripts were depleted and resulting libraries were minimally amplified and quantified for sequencing using a HiSeq4000 sequencer (Illumina, San Diego, CA) as previously described ${ }^{49}$. Transcriptomic sequencing data from each cell line was processed at AccuraScience LLC (Johnston, IA). Multidimensional sequencing data were visualized using principal component analysis charts.

Received: 29 July 2020; Accepted: 1 February 2021

Published online: 17 February 2021

\section{References}

1. Goldberg, J. L., Wax, M., Clark, A., \& Civan, M. M. In Ophthalmology (ed M. Yanoff, Duker, J. S.) Ch. Mechanisms of Glaucoma, 1018-1023 (Elsevier, 2019).

2. Bill, A. Some aspects of aqueous humour drainage. Eye (Lond) 7(Pt 1), 14-19. https://doi.org/10.1038/eye.1993.4 (1993).

3. Andres-Guerrero, V., Garcia-Feijoo, J. \& Konstas, A. G. Targeting Schlemm's canal in the medical therapy of glaucoma: current and future considerations. Adv. Ther. 34, 1049-1069. https://doi.org/10.1007/s12325-017-0513-z (2017).

4. Grant, W. M. Facility of flow through the trabecular meshwork. AMA Arch. Ophthalmol. 54, 245-248. https://doi.org/10.1001/ archopht.1955.00930020251012 (1955).

5. Grant, W. M. Further studies on facility of flow through the trabecular meshwork. AMA Arch. Ophthalmol. 60, 523-533. https:// doi.org/10.1001/archopht.1958.00940080541001 (1958).

6. Tamm, E. R. The trabecular meshwork outflow pathways: structural and functional aspects. Exp. Eye Res. 88, 648-655. https://doi. org/10.1016/j.exer.2009.02.007 (2009).

7. VanBuskirk, E. M. \& Grant, W. M. Influence of temperature and the question of involvement of cellular metabolism in aqueous outflow. Am. J. Ophthalmol. 77, 565-572. https://doi.org/10.1016/0002-9394(74)90472-3 (1974).

8. Bermudez, J. Y., Montecchi-Palmer, M., Mao, W. \& Clark, A. F. Cross-linked actin networks (CLANs) in glaucoma. Exp. Eye Res. 159, 16-22. https://doi.org/10.1016/j.exer.2017.02.010 (2017).

9. Clark, A. F. et al. Dexamethasone alters F-actin architecture and promotes cross-linked actin network formation in human trabecular meshwork tissue. Cell Motil. Cytoskelet. 60, 83-95. https://doi.org/10.1002/cm.20049 (2005).

10. Clark, A. F. et al. Glucocorticoid-induced formation of cross-linked actin networks in cultured human trabecular meshwork cells. Invest. Ophthalmol. Vis. Sci. 35, 281-294 (1994).

11. Polansky, J. R., Weinreb, R. N., Baxter, J. D. \& Alvarado, J. Human trabecular cells. I. Establishment in tissue culture and growth characteristics. Invest. Ophthalmol. Vis. Sci. 18, 1043-1049 (1979).

12. Polansky, J. R., Fauss, D. J. \& Zimmerman, C. C. Regulation of TIGR/MYOC gene expression in human trabecular meshwork cells. Eye (Lond) 14(Pt 3B), 503-514. https://doi.org/10.1038/eye.2000.137 (2000).

13. Keller, K. E. et al. Consensus recommendations for trabecular meshwork cell isolation, characterization and culture. Exp. Eye Res. 171, 164-173. https://doi.org/10.1016/j.exer.2018.03.001 (2018).

14. Stamer, W. D., Roberts, B. C., Howell, D. N. \& Epstein, D. L. Isolation, culture, and characterization of endothelial cells from Schlemm's canal. Invest. Ophthalmol. Vis. Sci. 39, 1804-1812 (1998).

15. Vahabikashi, A. et al. Increased stiffness and flow resistance of the inner wall of Schlemm's canal in glaucomatous human eyes. Proc. Natl. Acad. Sci. USA https://doi.org/10.1073/pnas.1911837116 (2019).

16. McDonnell, F., Dismuke, W. M., Overby, D. R. \& Stamer, W. D. Pharmacological regulation of outflow resistance distal to Schlemm's canal. Am. J. Physiol. Cell Physiol. 315, C44-C51. https://doi.org/10.1152/ajpcell.00024.2018 (2018).

17. Grant, W. M. Experimental aqueous perfusion in enucleated human eyes. Arch. Ophthalmol. 69, 783-801 (1963).

18. Overby, D. R., Stamer, W. D. \& Johnson, M. The changing paradigm of outflow resistance generation: towards synergistic models of the JCT and inner wall endothelium. Exp. Eye Res. 88, 656-670. https://doi.org/10.1016/j.exer.2008.11.033 (2009).

19. Rosenquist, R., Epstein, D., Melamed, S., Johnson, M. \& Grant, W. M. Outflow resistance of enucleated human eyes at two different perfusion pressures and different extents of trabeculotomy. Curr. Eye Res. 8, 1233-1240. https://doi.org/10.3109/027136889090139 02 (1989).

20. Carreon, T., van der Merwe, E., Fellman, R. L., Johnstone, M. \& Bhattacharya, S. K. Aqueous outflow-a continuum from trabecular meshwork to episcleral veins. Prog. Retin. Eye Res. 57, 108-133. https://doi.org/10.1016/j.preteyeres.2016.12.004 (2017).

21. Maepea, O. \& Bill, A. Pressures in the juxtacanalicular tissue and Schlemm's canal in monkeys. Exp. Eye Res. 54, 879-883 (1992).

22. Minckler, D. S., Baerveldt, G., Alfaro, M. R. \& Francis, B. A. Clinical results with the Trabectome for treatment of open-angle glaucoma. Ophthalmology 112, 962-967. https://doi.org/10.1016/j.ophtha.2004.12.043 (2005).

23. Van Buskirk, E. M. Trabeculotomy in the immature, enucleated human eye. Invest. Ophthalmol. Vis. Sci. 16, 63-66 (1977).

24. Grieshaber, M. C., Peckar, C., Pienaar, A., Koerber, N. \& Stegmann, R. Long-term results of up to 12 years of over 700 cases of viscocanalostomy for open-angle glaucoma. Acta Ophthalmol. 93, 362-367. https://doi.org/10.1111/aos.12513 (2015).

25. Grieshaber, M. C., Pienaar, A., Olivier, J. \& Stegmann, R. Canaloplasty for primary open-angle glaucoma: long-term outcome. Br. J. Ophthalmol. 94, 1478-1482. https://doi.org/10.1136/bjo.2009.163170 (2010). 
26. Krasnov, M. M. Microsurgery of glaucoma. Indications and choice of techniques. Am. J. Ophthalmol. 67, 857-864. https://doi. org/10.1016/0002-9394(69)90079-8 (1969).

27. Nesterov, A. P. Role of the blockade of Schlemm's canal in pathogenesis of primary open-angle glaucoma. Am. J. Ophthalmol. 70, 691-696. https://doi.org/10.1016/0002-9394(70)90484-8 (1970).

28. Ascher, K. W. The Aqueous Veins: Biomicroscopic Study of Aqueous Humor Elimination (Charles C. Thomas, Springfield, 1961).

29. Johnstone, M., Martin, E. \& Jamil, A. Pulsatile flow into the aqueous veins: manifestations in normal and glaucomatous eyes. Exp. Eye Res. 92, 318-327. https://doi.org/10.1016/j.exer.2011.03.011 (2011).

30. Kleinert, H. W. The compensation maximum; a new glaucoma sign in aqueous veins. AMA Arch. Ophthalmol. 46, 618-624 (1951).

31. Stambaugh, J. L., Fuhs, J. C. \& Ascher, K. W. Study of the compensation-maximum test on aqueous veins. AMA Arch. Ophthalmol. 51, 24-31 (1954).

32. Duffy, L. \& O’Reilly, S. Functional implications of cross-linked actin networks in trabecular meshwork cells. Cell. Physiol. Biochem. 45, 783-794. https://doi.org/10.1159/000487170 (2018).

33. Perkumas, K. M. \& Stamer, W. D. Protein markers and differentiation in culture for Schlemm's canal endothelial cells. Exp. Eye Res. 96, 82-87. https://doi.org/10.1016/j.exer.2011.12.017 (2012).

34. Vanden Hoek, T. L., Goossens, W. \& Knepper, P. A. Fluorescence-labeled lectins, glycoconjugates, and the development of the mouse AOP. Invest. Ophthalmol. Vis. Sci. 28, 451-458 (1987).

35. Kizhatil, K., Ryan, M., Marchant, J. K., Henrich, S. \& John, S. W. Schlemm's canal is a unique vessel with a combination of blood vascular and lymphatic phenotypes that forms by a novel developmental process. PLoS Biol. 12, e1001912. https://doi.org/10.1371/ journal.pbio.1001912 (2014).

36. Hamanaka, T., Bill, A., Ichinohasama, R. \& Ishida, T. Aspects of the development of Schlemm's canal. Exp. Eye Res. 55, 479-488. https://doi.org/10.1016/0014-4835(92)90121-8 (1992).

37. Dautriche, C. N. et al. A biomimetic Schlemm's canal inner wall: a model to study outflow physiology, glaucoma pathology and high-throughput drug screening. Biomaterials 65, 86-92. https://doi.org/10.1016/j.biomaterials.2015.06.034 (2015).

38. Cortes, F., Debacker, C., Peault, B. \& Labastie, M. C. Differential expression of KDR/VEGFR-2 and CD34 during mesoderm development of the early human embryo. Mech. Dev. 83, 161-164. https://doi.org/10.1016/s0925-4773(99)00030-1 (1999).

39. Miettinen, M., Rikala, M. S., Rys, J., Lasota, J. \& Wang, Z. F. Vascular endothelial growth factor receptor 2 as a marker for malignant vascular tumors and mesothelioma: an immunohistochemical study of 262 vascular endothelial and 1640 nonvascular tumors. Am. J. Surg. Pathol. 36, 629-639. https://doi.org/10.1097/PAS.0b013e318243555b (2012).

40. Hann, C. R., Bentley, M. D., Vercnocke, A., Ritman, E. L. \& Fautsch, M. P. Imaging the aqueous humor outflow pathway in human eyes by three-dimensional micro-computed tomography (3D micro-CT). Exp. Eye Res. 92, 104-111. https://doi.org/10.1016/j. exer.2010.12.010 (2011).

41. Kagemann, L. et al. Visualization of the conventional outflow pathway in the living human eye. Ophthalmology 119, 1563-1568. https://doi.org/10.1016/j.ophtha.2012.02.032 (2012).

42. Vascotto, S. G. \& Griffith, M. Localization of candidate stem and progenitor cell markers within the human cornea, limbus, and bulbar conjunctiva in vivo and in cell culture. Anat. Rec. A Discov. Mol. Cell Evol. Biol. 288, 921-931. https://doi.org/10.1002/ ar.a.20346 (2006).

43. Chowdhury, U. R., Jea, S. Y., Oh, D. J., Rhee, D. J. \& Fautsch, M. P. Expression profile of the matricellular protein osteopontin in primary open-angle glaucoma and the normal human eye. Invest. Ophthalmol. Vis. Sci. 52, 6443-6451. https://doi.org/10.1167/ iovs.11-7409iovs.11-7409[pii] (2011).

44. Stamer, W. D., Seftor, R. E., Williams, S. K., Samaha, H. A. \& Snyder, R. W. Isolation and culture of human trabecular meshwork cells by extracellular matrix digestion. Curr. Eye Res. 14, 611-617 (1995).

45. Chowdhury, U. R. et al. ATP-sensitive potassium (KATP) channel activation decreases intraocular pressure in the anterior chamber of the eye. Invest. Ophthalmol. Vis. Sci. 52, 6435-6442. https://doi.org/10.1167/iovs.11-7523iovs.11-7523 (2011).

46. O'Reilly, S. et al. Inducers of cross-linked actin networks in trabecular meshwork cells. Invest. Ophthalmol. Vis. Sci. 52, 7316-7324. https://doi.org/10.1167/iovs.10-6692 (2011).

47. Roy Chowdhury, U., Bahler, C. K., Holman, B. H. \& Fautsch, M. P. ATP-sensitive potassium (KATP) channel openers diazoxide and nicorandil lower intraocular pressure by activating the Erk1/2 signaling pathway. PLoS ONE 12, e0179345. https://doi.org/10.1371/ journal.pone.0179345 (2017).

48. Fautsch, M. P., Bahler, C. K., Jewison, D. J. \& Johnson, D. H. Recombinant TIGR/MYOC increases outflow resistance in the human anterior segment. Invest. Ophthalmol. Vis. Sci. 41, 4163-4168 (2000).

49. Wieben, E. D. et al. Trinucleotide repeat expansion in the transcription factor 4 (TCF4) gene leads to widespread mRNA splicing changes in fuchs' endothelial corneal dystrophy. Invest. Ophthalmol. Vis. Sci. 58, 343-352. https://doi.org/10.1167/iovs.16-20900 (2017).

\section{Acknowledgements}

We would like to thank Justin Li, PhD at AccuraScience LLC for his helpful suggestions in processing our RNA Sequencing data.

\section{Author contributions}

U.R.C. and M.P.F. were involved in the experimental design, data analysis and manuscript writing. U.R.C., C.K.B., C.R.H. and B.H.H. performed technical aspects of cell line culture and histology. U.R.C. designed figures and tables. C.K.B. and C.R.H. edited manuscript prior to submission.

\section{Competing interests}

The authors declare no competing interests.

\section{Additional information}

Supplementary Information The online version contains supplementary material available at https://doi. org/10.1038/s41598-021-83558-6.

Correspondence and requests for materials should be addressed to U.R.C.

Reprints and permissions information is available at www.nature.com/reprints.

Publisher's note Springer Nature remains neutral with regard to jurisdictional claims in published maps and institutional affiliations. 
(c) (i) Open Access This article is licensed under a Creative Commons Attribution 4.0 International cc) License, which permits use, sharing, adaptation, distribution and reproduction in any medium or format, as long as you give appropriate credit to the original author(s) and the source, provide a link to the Creative Commons licence, and indicate if changes were made. The images or other third party material in this article are included in the article's Creative Commons licence, unless indicated otherwise in a credit line to the material. If material is not included in the article's Creative Commons licence and your intended use is not permitted by statutory regulation or exceeds the permitted use, you will need to obtain permission directly from the copyright holder. To view a copy of this licence, visit http://creativecommons.org/licenses/by/4.0/.

(C) The Author(s) 2021 